\title{
Papel de la ecografia en la valoración del resultado del tratamiento endoscópico del RVU
}

\author{
Serrano Durba A, Bonillo García MA, Domínguez Hinarejos C, Sanguesa C*, \\ Muro D, García Ibarra F.
}

Servicio de Urología y *Radiología Infantil. Hospital La Fe. Valencia.

Actas Urol Esp 2006; 30 (4): 402-405

\section{RESUMEN}

PAPEL DE LA ECOGRAFÍA EN LA VALORACIÓN DEL RESULTADO DEL TRATAMIENTO ENDOSCÓPICO DEL RVU Introducción: Desde el comienzo del tratamiento endoscópico del reflujo vesicoureteral (RVU) se protocolizó el uso de la ecografia basándose en dos indicaciones: a) Detección de las complicaciones (ectasia); b) Evaluación del resultado (visualización del material implantado y la presencia del "jet ureteral" mediante ecodoppler-color).

Objetivos: Determinar el valor de la ecografia en la evaluación del resultado del tratamiento endoscópico del RVU y en la detección de sus complicaciones.

Material y métodos: Durante el periodo 2001-2002 aplicamos el tratamiento endoscópico a un total de 261 unidades ureterales de todo grado y etiología. 246 evaluadas postoperatoriamente con ecografia al mes y todos con cistografia a los tres meses. En una muestra aleatoria de 92 unidades se ha comparado el resultado de la cistografia isotópica con la visualización del material implantado en la vejiga, y en una muestra aleatoria de 56 unidades se ha comparado el resultado de la cistografia isotópica con la presencia de jet ureteral en el estudio con ecodoppler-color tras hidratación del paciente. Para ello se han realizado las correspondientes tablas comparativas 2 × 2 y se ha calculado la Sensibilidad (S) y Especificidad (E) de las pruebas, así como sus valores predictivo positivo (VPP) y negativo (VPN), el grado de acuerdo de ambas pruebas con el indice de Kappa y el nivel de significación estadística con Chi cuadrado.

Resultados: La ecografia resultó normal o sin cambios respecto a estudios previos en 213 unidades (86,58\%) y en 5 (2,03\%) ha aparecido una ectasia atribuible al tratamiento, de las cuales 3 han cursado con dolor renoureteral y en los otros 2 ha desaparecido espontáneamente en controles posteriores. La S y E de la presencia de material implantado ha sido de 94\% y 10\% respectivamente y sus VPP y VPN de $80 \%$ y $10 \%$ y ésta tiene un nivel de significación estadística del 0,564 y un grado de correlación de 0,0675. La S y E de la visualización del jet ha sido de $82 \%$ y $30 \%$, con VPP de $84 \%$ y VPN de 27, un nivel de significación estadística del 0,836 y un grado de correlación de 0,121 .

Conclusiones: La ecografia no es una técnica útil en la evaluación del resultado del tratamiento endoscópico del RVU. Su papel en la evaluación de las complicaciones es muy reducido y solamente está indicada para confirmar la sospecha de ectasia en aquellos pacientes que presentan clínica de dolor renoureteral.

Palabras clave: Reflujo. Tratamiento endoscópico. Ecografia. Complicaciones.

\section{ABSTRACT}

ULTRASOUND ROLE IN ASSESSMENT OF THE VESICOURETERIC REFLUX ENDOSCOPIC TREATMENT RESULTS

Introduction: Since the beginning of the application of the RVU treatment, the use of the ultrasound was used taking into account two main indications/symptoms: a) identification of the complications; b) assessment of the result (visualization of the implanted material and the presence of the "uretheral jet" by means or through/of ecodoppler-color).

Objective: To determine the value of the ultrasound in the assessment of the result of the endoscopic treatment of the RVU and in the detection of its complications.

Material and method: From 2001-2002 we have carried out the endoscopic treatment to an overall of 261 ureteral units of all degree and etiology. 246 were assessed post-operation with an ultrasound per month and all of them were evaluated with a cistography after 3 months. In a random sample of 92 units, the result of the isotopic cistography has been compared with the visualization of the implanted material in the bladder; and in a random sample of 56 units, the result of the isotopic cistography has been compared with the presence of ureteral jet in the study with ecodoppler-color after moisturizing of the patient. In order to do, we have done two corresponding comparative charts $2 \times 2$ and we have calculated the Sensibility and Specificity of the tests, as well as their predictive positive and negative values, the degree of similarity of both tests with the Kappa index, and the degree of statistical relevance with Chi squared.

Results: The ultrasound did not show significant changes if compared to previous studies in 213 units $(86,58 \%)$ although in 5 patients an ectasia has been identified as attributed to the treatment. 3 of this patients have developed renoureteral pain, and in 2 pain has spontaneously disappeared as shown in later tests. The $\mathrm{S}$ and the $\mathrm{E}$ of the presence of implanted material is $94 \%$ and $10 \%$ respectively and its VPP and VPN of $80 \%$ and $10 \%$ and estadistical. The S and $\mathrm{E}$ of the jet visualization is $82 \%$ and $30 \%$, with a VPP of $84 \%$ and a VPN of $27 \%$, a level of statistical relevance of 0.836 and a degree of correlation of 0.121 .

Conclusions: Ultrasound is not a useful technique in the assessment of the result of the endoscopic treatment of the RVU. The role it plays in the assessment of complications is a small one and its use is only indicated to confirm the suspicion of ectasia in those patients that present renoureteral pain

Keywords: Reflux. Endoscopic treatment. Ultrasound. Complications. 
$\mathrm{E}^{1}$ reflujo vesicoureteral (RVU) primario es la anomalía urológica más frecuente en el niño que afecta al $1 \%$ de los mismos ${ }^{1,2}$. Se detecta en el $30-50 \%$ de aquellos afectos de una infección del tracto urinario (ITU) ${ }^{3,4}$, combinación que predispone al daño del parénquima renal, estimándose que la nefropatía secundaria al RVU es causa de la insuficiencia renal terminal en el 3$25 \%$ de los niños y en el 10-15\% de los adultos ${ }^{5}$.

Desde su descripción al principio de la década de los $80^{3}$, la inyección de agentes inertes se ha convertido en una alternativa al tratamiento clásico del RVU (cirugía abierta y al tratamiento conservador mediante la profilaxis antibiótica a largo plazo) ${ }^{6}$. Al igual que el reimplante ureteral, el tratamiento endoscópico ofrece un alto porcentaje de resolución inmediata del RVU con la diferencia de que este último es un procedimiento mínimamente invasivo que evita el trauma de la cirugía abierta acortando la hospitalización y recuperación del paciente ${ }^{7}$.

A pesar de que la prueba diagnóstica por excelencia para demostrar la resolución del RVU es la cistografia isotópica, tradicionalmente, la evaluación de los resultados obtenidos tras la inyección de agentes ha justificado la realización de un control ecográfico en el seguimiento de estos pacientes, siendo las principales variables a estudiar, la visualización del material implantado y las características fisicas del jet ureteral medido mediante ecodoppler-color. Del mismo modo, la ecografia se realizaba de forma protocolizada con la intención de detectar la presencia de complicaciones asociadas ${ }^{8}$.

Nuestro objetivo es determinar el valor del estudio ecográfico en la evaluación del resultado del tratamiento endoscópico del RVU y en la detección de sus complicaciones.

\section{MATERIAL Y MÉTODO}

En el periodo entre 1999-2001 se trataron endoscópicamente en nuestro servicio 261 unidades ureterales en un grupo de 174 pacientes (119 niñas/ 55 niños). En el momento del procedimiento los pacientes tenían una edad media de 7,9 años (15m-16a). Se incluyeron en el estudio tanto RVU primarios como secundarios al igual que tampoco se excluyó a ningún paciente por el grado de RVU. Para la inyección endoscópica utilizamos un cistoscopio de $9,5 \mathrm{Ch}$, una aguja de
Williams de 5Fr y bajo anestesia general tras la identificación del orificio ureteral correspondiente, un mismo urólogo infantil procede a la inyección submeática de polidimetilxilosano (volumen medio de $0,72 \mathrm{ml}$ ), según la técnica descrita por Puri ${ }^{9}$.

A 246 de los pacientes se les realiza un control ecográfico al mes y una cistografía isotópica al tercer mes de la intervención. Comparamos en una muestra aleatoria de 92 unidades ureterales el resultado de la cistografía isotópica con los resultados de la ecografia, en concreto la visualización del material implantado en la vejiga, y en una muestra aleatoria de 56 unidades el resultado de la cistografía isotópica con la presencia de jet ureteral en el estudio con ecodoppler-color tras hidratación del paciente. Para ello se han realizado las correspondientes tablas de contingencia $2 \times 2$ y se ha calculado la Sensibilidad (S) y Especificidad $(E)$ de las pruebas, así como sus valores predictivo positivo (VPP) y negativo (VPN), el grado de acuerdo de ambas pruebas con el índice de Kappa $(k)$ y el nivel de significación estadística $(p)$ con Chi cuadrado $\left(C h i^{2}\right)$.

\section{RESULTADOS}

De las 246 unidades ureterales evaluadas, en $241(97,97 \%)$ no se objetivó la presencia de complicaciones asociadas. En cambio, en 5 (2,03\%) se detectó una ectasia ureteropielocalicial mayor a la constatada previa a la intervención o de aparición de novo. Dos de estos pacientes no presentaron sintomatología alguna y fue un hallazgo casual, sin embargo, en los otros 3 pacientes encontramos que durante el seguimiento habían sufrido una clínica compatible con crisis renoureteral, de los que 2 de ellos precisaron la realización de una nefrostomía percutánea tras imposibilidad de cateterización. En estos 2 pacientes se le realizó un reimplante ureteral según la técnica descrita por Cohen. La ectasia del resto de los pacientes se resolvió espontáneamente en el transcurso del seguimiento clínico.

En cuanto al estudio estadístico, cuando comparamos el resultado de la cistografía isotópica con la visualización del material implantado en la vejiga (Fig. 1), observamos que conseguimos una $\mathrm{S}$ del $94 \%$ con un VPP del 80\% y una E y VPN del $10 \%$, y que ésta tiene un nivel de significación estadística del 0,564 y un grado de correlación de 


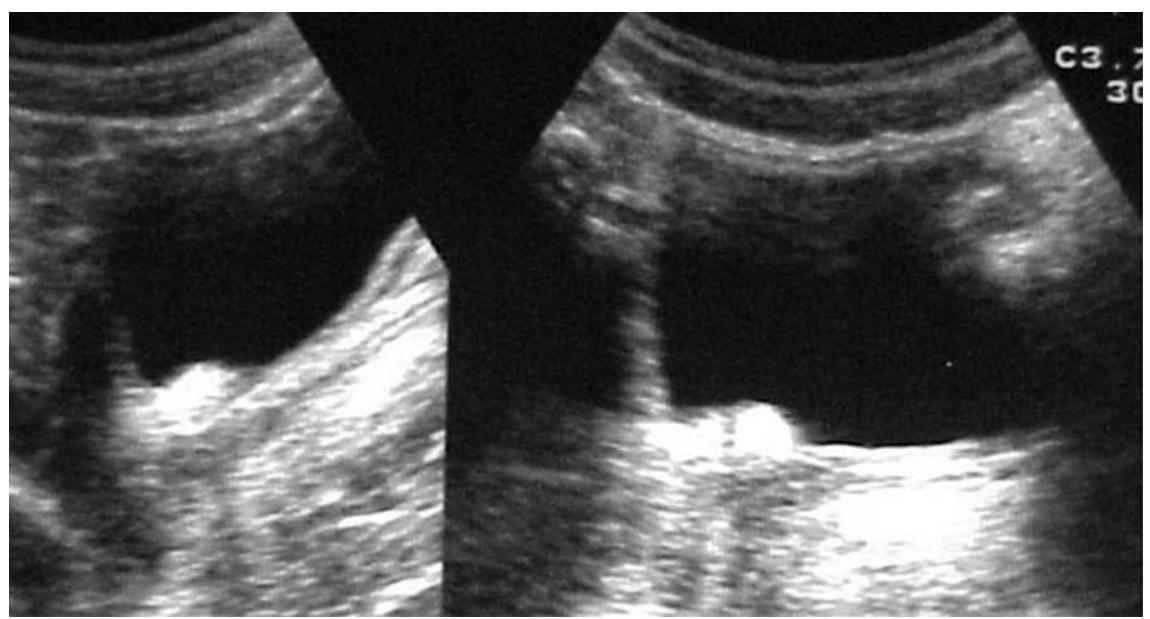

FIGURA 1. Visualización ecográfica del material inyectado como imagen hiperecoica en el interior de la vejiga.

0,0675. Por el contrario, el resultado de la cistografía isotópica comparado con la presencia de jet ureteral en el estudio con ecodoppler-color tras hidratación del paciente (Fig. 2), alcanza una S del 82\% con un VPP del 84\% y una E del 30\% con un VPN del 27\%, y que ésta tiene un nivel de significación estadística del 0,836 y un grado de correlación de 0,121 .

\section{DISCUSIÓN}

La inyección endoscópica de agentes inertes es una alternativa adecuada al reimplante ureteral mediante cirugía abierta, al ser una técnica mínimamente invasiva con unos resultados de curación aceptables en función de la sustancia utilizada. El seguimiento de estos pacientes está

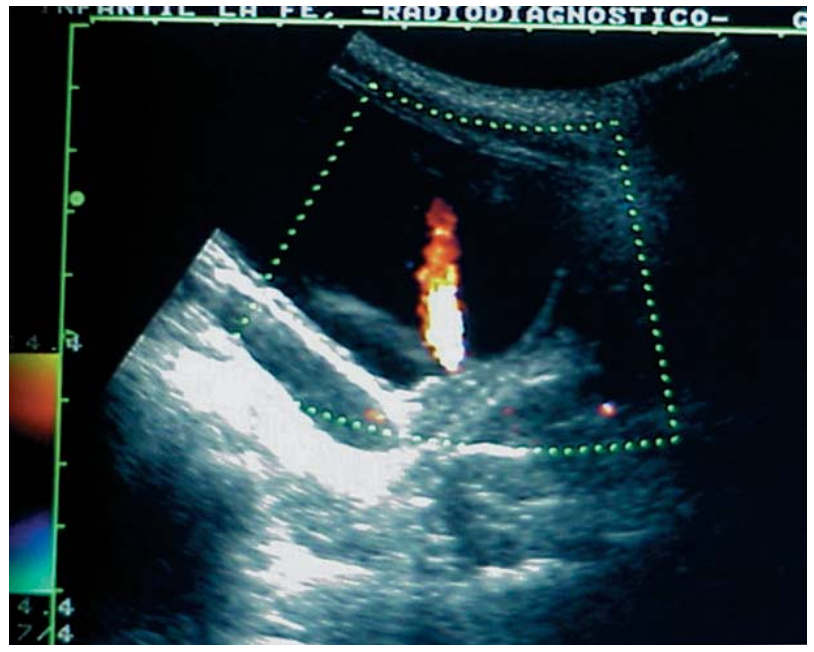

FIGURA 2. Presencia del jet ureteral en la eco dopplercolor. marcado por dos circunstancias, la aparición de complicaciones inherentes a la técnica y la presencia de RVU. Para demostrar la curación del RVU y su estabilidad en el tiempo se realizaban cistografias incluso en un principio en la misma mesa de quirófano. Con el fin de obviar el número de veces que había que irradiar al paciente, algunos autores propusieron la realización de un control ecográfico y establecieron una correlación entre la presencia del material inyectado con la curación del RVU. Así Puri ${ }^{10}$ afirma que la visualización del habón en la ecografía indicaba ausencia de reflujo. Bhatti ${ }^{11}$ en un estudio realizado en 135 pacientes a los que les inyecta Teflón, relaciona la ausencia de habón en la ecografía con la existencia de RVU, decidiendo la necesidad de una nueva intervención basada en estos hallazgos, sin tener que realizar una cistografia previa. En este sentido, otros autores en una publicación más reciente constatan el fracaso terapéutico con la ausencia o irregularidad del material inyectado ${ }^{12}$.

En nuestro trabajo, sin embargo, no hemos encontrado dicha correlación y al igual que en el trabajo realizado por Gómez Fraile ${ }^{13}$, la ausencia de habón en el estudio ecográfico no se correlacionaba con la existencia de reflujo ni la presencia del material hiperecoico la resolución del mismo. Tampoco ha sido posible constatar la relación existente entre las características físicas del jet ureteral medido mediante Eco-Doppler y la curación del RVU.

Con relación a la detección de complicaciones, solamente en 5 casos (2,03\%) la ecografía encontró hallazgos, es decir, en el 97,97\%, la Eco fue absolutamente innecesaria y de estos cinco casos con complicaciones, todos los asintomáticos presentaron una resolución espontánea completa, por lo que en ellos tampoco hubiera sido necesaria. Además, todos los casos que precisaron de alguna maniobra terapéutica habían presentado clínica, con lo cual la Eco sólo sirvió para corroborar la sospecha ante la presencia de manifestaciones clínicas. 


\section{CONCLUSIONES}

La ecografía no es una técnica útil en la evaluación del resultado del tratamiento endoscópico del RVU. Su papel en la detección de las complicaciones es muy reducido y solamente está indicada para confirmar la sospecha de ectasia en aquellos pacientes que presentan clínica de dolor renoureteral.

\section{REFERENCIAS}

1. Jacobson SH, Hansson S, Jakobsson B. Vesico-ureteric reflux: occurrence and long-term risks. Acta Paediatr Suppl 1999;88(431):22-30.

2. Smellie JM, Barratt TM, Chantler C, Gordon I, Prescod NP, Ransley PG, et al. Medical versus surgical treatment in children with severe bilateral vesicoureteric reflux and bilateral nephropathy: a randomised trial. Lancet 2001;357(9265): 1329-1333.

3. Chertin B, Colhoun E, Velayudham M, Puri P. Endoscopic treatment of vesicoureteral reflux: 11 to 17 years of followup. J Urol 2002;167(3):1443-5; discussion 1445-1446.

4. Elder JS. Guidelines for consideration for surgical repair of vesicoureteral reflux. Curr Opin Urol 2000;10(6):579-585.

5. Bailey RR. Vesico-ureteric reflux and reflux nephropathy. Kidney Int Suppl 1993;42:S80-S85.

6. Puri P, Chertin B, Velayudham M, Dass L, Colhoun E. Treatment of vesicoureteral reflux by endoscopic injection of dextranomer/hyaluronic Acid copolymer: preliminary results. J Urol 2003;170(4 Pt 2):1541-1544; discussion 1544.

7. Serrano A, Estornell F, Domínguez C, M M, F G. Eficacia del tratamiento endoscópico del reflujo vesicoureteral en la infancia con polidimetilxilosano. Urol Intergr Invest 2002; 7(4):490-493.
8. Rypens F, Avni EF, Bank WO, Schulman CC, Struyven J. The ureterovesical junction in children: sonographic findings after surgical or endoscopic treatment. AJR Am J Roentgenol 1992;158(4):837-842.

9. Puri P, O'Donnell B. Correction of experimentally produced vesicoureteric reflux in the piglet by intravesical injection of Teflon. Br Med J (Clin Res Ed) 1984;289(6436):5-7.

10. Puri P. Ten year experience with subureteric Teflon (polytetrafluoroethylene) injection (STING) in the treatment of vesico-ureteric reflux. Br J Urol 1995;75(2):126-131.

11. Bhatti HA, Khattak H, Boston VE. Efficacy and causes of failure of endoscopic subureteric injection of Teflon in the treatment of primary vesicoureteric reflux. $\mathrm{Br} \mathrm{J}$ Urol 1993;71(2):221-225.

12. Paltiel HJ, Diamond DA, Zurakowski D, Drubach LA, Atala A. Endoscopic treatment of vesicoureteral reflux with autologous chondrocytes: postoperative sonographic features. Radiology 2004;232(2):390-397.

13. Gomez Fraile A, Suarez Charnenco A, Aransay Bramtot A, Lopez Vazquez F, Serrano C. Estudio del valor diagnóstico de la visualización ecográfica del material inyectado en el tratamiento endoscópico del reflujo vesicoureteral. Cir Pediatr 2004;17(4):185-188.

Dr. A. Serrano Durba

Servicio de Urología

Hospital La Fe

Avda. Campanar, 21

46009 Valencia

E-mail: miggs89@yahoo.es

(Trabajo recibido el 13 de abril de 2005) 